\title{
Thermal conductivity of $\mathrm{Tl}_{2} \mathrm{Ba}_{2} \mathrm{Ca}_{2} \mathrm{Cu}_{3} \mathrm{O}_{10}$ ceramics from $300 \mathrm{~K}$ down to $0.1 \mathrm{~K}$
}

\author{
C. Uher, S.D. Peacor and J. Shewchun \\ Department of Physics, University of Michigan, Ann Arbor, Michigan 48109, USA
}

Received 11 February 1991

Revised manuscript received 29 March 1991

\begin{abstract}
Thermal conductivity, $\kappa$, of two ceramic samples of $\mathrm{Tl}_{2} \mathrm{Ba}_{2} \mathrm{Ca}_{2} \mathrm{Cu}_{3} \mathrm{O}_{10}$ has been measured over a ter yerature range from $300 \mathrm{~K}$ down to $0.1 \mathrm{~K}$. At high temperatures, the data show features similar to the thermal conductivity of $\mathrm{Y}-\mathrm{Ba}-\mathrm{Cu}-\mathrm{O}$ and $\mathrm{Bi}-\mathrm{Sr}-\mathrm{Ca}-$ $\mathrm{Cu}-\mathrm{O}$ ceramics in both the magnitude and the temperature dependence. Specifically, a sudden increase in the thermal conductivity is observed at the onset of a superconducting transition near $120 \mathrm{~K}$ culminating in a pronounced maximum of $\kappa$ around $75 \mathrm{~K}$ and an eventual rapid decrease of the thermal conductivity at lower temperatures. From $5 \mathrm{~K}$ down to $0.1 \mathrm{~K}$ we observe the thermal conductivity to decrease with an average power law exponent between 2.4 and 2.5. Such a temperature dependence is comparable with that for sintered $\mathrm{Bi}-\mathrm{Sr}-\mathrm{Ca}-\mathrm{Cu}-\mathrm{O}$ samples, but differs from the quadratic variation typical for $\mathrm{Bi}-\mathrm{Sr}-\mathrm{Ca}-\mathrm{Cu}-\mathrm{O}$ single crystals and the $T$-linear asymptotic behavior characteristic of $\mathrm{Y}-\mathrm{Ba}-\mathrm{Cu}-\mathrm{O}$ and $\mathrm{La}-\mathrm{Sr}-\mathrm{Cu}-\mathrm{O}$ ceramics.
\end{abstract}

Superconductivity in Tl-based perovskite was discovered early in 1988 by Sheng and Hermann [1] and, till this day, these compounds represent high- $T_{\mathrm{c}}$ materials with the highest reproducible transition temperature, $T_{\mathrm{c}} \sim 125 \mathrm{~K}$. In its more usual double $\mathrm{Tl}$ layer form the structure of $\mathrm{Tl}-\mathrm{Ba}-\mathrm{Ca}-\mathrm{Cu}-\mathrm{O}$ compounds is similar to those of $\mathrm{Bi}-\mathrm{Sr}-\mathrm{Ca}-\mathrm{Cu}-\mathrm{O}$ superconductors and can be designated as $\mathrm{Tl}_{2} \mathrm{Ba}_{2} \mathrm{Ca}_{n-1} \mathrm{Cu}_{n} \mathrm{O}_{2 n+4}$. The index $n$ indicates the number of $\mathrm{Cu}-\mathrm{O}$ planes sandwiched between the $\mathrm{Tl}$ layers and has a profound effect on superconducting properties of this material. As $n$ increases, the structure progresses from a Ca-free (2201) phase with a somewhat uncertain superconducting transition temperature reported [2] to lie between 6 and $80 \mathrm{~K}$, to a (2212) superconductor with $T_{\mathrm{c}}$ near $106 \mathrm{~K}$ and, eventually, to a (2223) material that superconducts up to $125 \mathrm{~K}$. It is interesting to note that the double Tl layer arrangement is not necessarily required to achieve high values of $T_{\mathrm{c}}$. Among single $\mathrm{Tl}$ layer compounds discovered by Parkin et al. [3], is the (1234) phase which was identified by Ihara et al. [4] as a $120 \mathrm{~K}$ superconductor. Numerous other structural modifications have been discovered which at- test to the difficulty of preparing single-phase samples in this family of perovskite.

Although Tl-based perovskite superconductors are appealing for their high transition temperature, reports on their thermal transport properties are few. The lack of interest undoubtedly stems from hesitancy to handle this rather toxic compound. In this paper we report on our investigations of the thermal conductivity of a nominally (2223) phase of Tl-based ceramics covering a wide range of temperatures.

Samples used in these measurements were prepared in-house following a sintering recipe similar to the one described by Wu et al. [5]. Commercially available $\mathrm{CaCO}_{3}$ (99.999\%), $\mathrm{BaCO}_{3}$ (99.99\%), and $\mathrm{CuO}(99.999 \%)$ powders were mixed and ground in an alumina mortar. The mixture was then reacted in an alumina crucible in air at $925^{\circ} \mathrm{C}$, ground to a powder, and the process repeated once more. This $\mathrm{Ba}-\mathrm{Ca}-\mathrm{Cu}-\mathrm{O}$ precursor was formed first to avoid a prolonged exposure of $\mathrm{Tl}_{2} \mathrm{O}_{3}$ at the desired reaction temperature, where it decomposes rapidly, resulting in the loss of $\mathrm{Tl}$. The $\mathrm{Tl}_{2} \mathrm{O}_{3}(99.995 \%)$ was then added to the precursor, the mixture reground and pressed into a pellet at $40 \mathrm{kpsi}$. Typical pellet sizes were $12 \mathrm{~mm}$ in diameter and $1-2 \mathrm{~mm}$ thick. The fi- 
nal composite is formed by reacting the pellet inside a covered platinum crucible placed in a furnace with flowing oxygen and held at $895^{\circ} \mathrm{C}$ for about $5 \mathrm{~min}$. Quantities of various powders used were aimed at synthesizing the (2223) phase. X-ray analysis indicates that the (2223) phase is indeed the dominant component but a small admixture of other phases (at an approximately $5 \%$ level) is present as well. Typical grain size of our samples is in the range 5-10 $\mu \mathrm{m}$. Magnetization and the electrical resistivity measurements indicate the onset of a superconducting transition at about $121 \mathrm{~K}$.

Measurements of the thermal conductivity were carried out in a ${ }^{4} \mathrm{He}$ cryostat down to $2 \mathrm{~K}$ and extended to subkelvin temperatures in a dilution refrigerator. Samples were of sufficiently large size to allow the use of the classical steady-state method of measurement. Above $4 \mathrm{~K}$ we employed a pair of calibrated glassy carbon thermometers together with a differential thermocouple made from $\mathrm{Au}+0.07$ at. \% $\mathrm{Fe} /$ chromel $\mathrm{P}$ wires. At very low temperatures we used a pair of calibrated germanium resistance sensors. Samples were attached to a cold tip of the cryostat with the aid of Stycast epoxy. Details concerning the experimental set-up as well as a discussion pertaining to radiation losses can be found elsewhere [6].

The thermal conductivity of two samples of $\mathrm{Tl}_{2} \mathrm{Ba}_{2} \mathrm{Ca}_{2} \mathrm{Cu}_{3} \mathrm{O}_{10}$ cut from different disks is shown in fig. 1. For comparison, we show also the data from

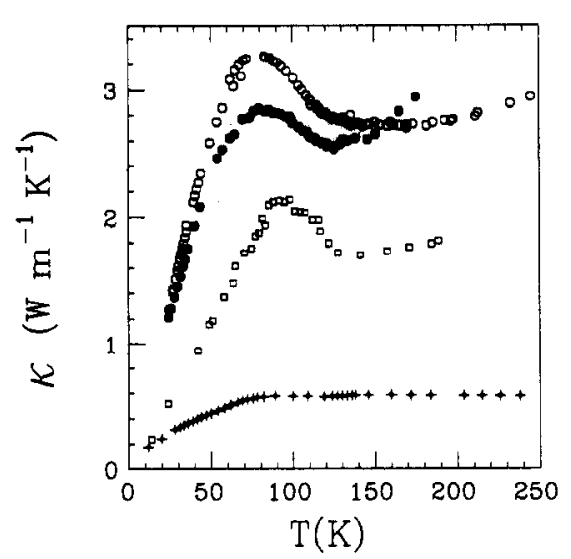

Fig. 1. Thermal conductivity of sintered samples of $\mathrm{Tl}_{2} \mathrm{Ba}_{2} \mathrm{Ca}_{2} \mathrm{Cu}_{3} \mathrm{O}_{10}$. The data of Aliev et al. [7] are represented by open squares, and the measurements of Castello et al. [8] by stars. the investigations of $\kappa$ by Aliev et al. [7] (depicted by squares) and by Castello et al. [8] (stars). We note that the magnitude of the thermal conductivity of Tl-based perovskites is comparable to those of other high- $T_{\mathrm{c}}$ ceramics [9] where, near $300 \mathrm{~K}$, the thermal conductivity lies typically between 1 and 10 $\mathrm{Wm}^{-1} \mathrm{~K}^{-1}$. Differences in the magnitude of the thermal conductivity of Tl-based samples shown in fig. 1 are undoubtedly associated with varying defect structures associated with sintered samples and are typical of a variation observed in all families of superconducting ceramics. From the electrical resistivity, see the inset in fig. 2, and using the WiedemannFranz law, we estimate that less than $5 \%$ of the total heat current is due to the flow of free charge carriers. Thus, the thermal conductivity is dominated by the phonons. As is the case of virtually all the data on sintered perovskites, the thermal conductivity increases with increasing temperaıure above $T_{\mathrm{c}}$. In view of a strong dominance of the phonon contribution to the heat flow, this is somewhat surprising. Well above $100 \mathrm{~K}$ one would expect to observe the influence of the phonon-phonon Umklapp processes and the ensuing $1 / T$-dependence of the thermal conductivity. Although a radiation heat leak may account for a part (but not all) of the rising heat conductivity with $T$, the fact remains that high-quality single crystals are a more suitable medium to resolve the phononphonon interaction clearly $[10,11]$.

The most dramatic feature of the data in fig. 1 is a sharply rising thermal conductivity below $120 \mathrm{~K}$ and a pronounced peak near $75 \mathrm{~K}$. The onset of the rise in $\kappa$ coincides with the samples becoming superconducting and, just as in the case of $\mathrm{Y}-\mathrm{Ba}-\mathrm{Cu}-$ $\mathrm{O}$ or $\mathrm{Bi}-\mathrm{Sr}-\mathrm{Ca}-\mathrm{Cu}-\mathrm{O}$ superconductors, we believe this phenomenon has its origin in an enhancement of the mean free path ( $\mathrm{mfp}$ ) of phonons as the free carriers (holes) condense. Since the charge carriers here contribute a small fraction of the total thermal conductivity, their withdrawal from the energy transport on their condensation into the Cooper sea does not lead to a precipitous decrease of the thermal conductivity as is the case in conventional superconductors. On the contrary, the inability of the Cooper pairs to scattering phonons brings about a sharply increasing phonon mfp which leads to a strong enhancement in the already dominant phonon thermal conductivity. As the temperature decreases 
the gain in the phonon thermal conductivity due to the mfp increase is eventually countered by freezing out of the phonon population and, as a consequence, a peak is observed on the thermal conductivity. While our data agree well with the investigations of Aliev et al. and of Jezowski et al. [12], the increase in the thermal conductivity below $T_{\mathrm{c}}$ in the measurements of Castello et al. is barely noticeable. This particular sample has a rather low value of $\kappa$ and it is likely that phonon scattering on impurities and/or other phases is strong enough to preclude any significant enhancement in the phonon $\mathrm{mfp}$ as the carriers condense below $T_{\mathrm{c}}$.

Temperature dependence of $\kappa$ below the maximum, particularly its asymptotic behavior at very low temperatures, is a controversial subject that is by no means settled. Very naively, at temperatures $T \ll T_{c}$, one would expect phonons to scatter on structural imperfections of the lattice, such as, e.g., grain boundaries, leading to a constant $\mathrm{mfp}$ and a $T^{3}$ boundary scattering regime of the thermal conductivity. With a notable exception [13] of single crystals of $\mathrm{La}_{2-x} \mathrm{Sr}_{x} \mathrm{CuO}_{4}$ where the temperature dependence is approximately $T^{2.6}$, the power-law exponents of $\kappa(T)$ at low temperature for all other high- $T_{\mathrm{c}}$ superconductors of either ceramic or single crystal forms have, in all cases [6], been measured as much smaller than 3. Furthermore, the estimated mfp of phonons is frequently substantially smaller than the typical grain size or the dimensions of the crystal, suggesting that some intragrain scattcring mechanism is important. A quadratic temperature dependence, which is often a good approximation of the experimental situation in ceramic samples over a limited temperature range around $10 \mathrm{~K}$, has led to the suggestion [14] that such an intragrain phonon scattering mechanism results from two-level tunneling states. This scenario is borrowed from the thermal conductivity of glasses $[15,16]$ where such twolevel tunneling entities are assumed to govern the heat transport. The $T^{2}$ dependence observed subsequently in the thermal conductivity of both single crystal $[17,18]$ and hot-pressed [19] $\mathrm{Bi}-\mathrm{Sr}-\mathrm{Ca}-\mathrm{Cu}-$ $\mathrm{O}$ superconductors down to below $0.1 \mathrm{~K}$ provided additional support for the tunneling model. Unfortunately, the quadratic temperature dependence of $k$ is not universal across the spectrum of high- $T_{\mathrm{c}}$ superconductors. For instance, ceramic forms of $\mathrm{Y}-\mathrm{Ba}-$
$\mathrm{Cu}-\mathrm{O}$ invariably indicate $[20-22]$ a crossover to a $T$-linear dependence of $\kappa(T)$ below about $0.5 \mathrm{~K}$ and a similar trend has been seen recently on single crystals of the same family of materials [18]. It has been speculated that this linear dependence of $\kappa(T)$ is associated with a small number of normal (uncondensed) carriers. Their contribution to the thermal conductivity overtakes the phonon term at sufficiently low temperatures. Because of the structural affinity between $\mathrm{Bi}-\mathrm{Sr}-\mathrm{Ca}-\mathrm{Cu}-\mathrm{O}$ and $\mathrm{Tl}-\mathrm{Ba}-\mathrm{Ca}-$ $\mathrm{Cu}-\mathrm{O}$ high- $T_{\mathrm{c}}$ perovskites, one would expect the lowtemperature behavior of Tl-based samples to be similar to that displayed by Bi-based superconductors. Although no data are available yet for either single crystals or hot-pressed samples of $\mathrm{Tl}-\mathrm{Ba}-\mathrm{Ca}-\mathrm{Cu}-\mathrm{O}$, a comparison between the sintered forms of $\mathrm{Bi}-\mathrm{Sr}-$ $\mathrm{Ca}-\mathrm{Cu}-\mathrm{O}$ and $\mathrm{Tl}-\mathrm{Ba}-\mathrm{Ca}-\mathrm{Cu}-\mathrm{O}$ indicates that the behaviour is, indeed, quite similar. As fig. 2 indicates, over a rather extended temperature range from $3 \mathrm{~K}$ down to $0.1 \mathrm{~K}$ both $\mathrm{Tl}-\mathrm{Ba}-\mathrm{Ca}-\mathrm{Cu}-\mathrm{O}$ samples display a non-integer power law dependence that is not too different from the $T^{2.3}$ variation observed in sintered $\mathrm{Bi}-\mathrm{Sr}-\mathrm{Ca}-\mathrm{Cu}-\mathrm{O}[19]$. We detect no weakening of the power law dependence at the lowest temperatures such as observed in $\mathrm{Y}-\mathrm{Ba}-\mathrm{Cu}-\mathrm{O}$ superconductors. Because of its steep temperature dependence, the thermal conductivity of $\mathrm{Tl}-\mathrm{Ba}-\mathrm{Ca}-$ $\mathrm{Cu}-\mathrm{O}$ samples below $1 \mathrm{~K}$ is nearly an order of magnitude smaller than those of $\mathrm{Y}-\mathrm{Ba}-\mathrm{Cu}-\mathrm{O}$ ceramics.

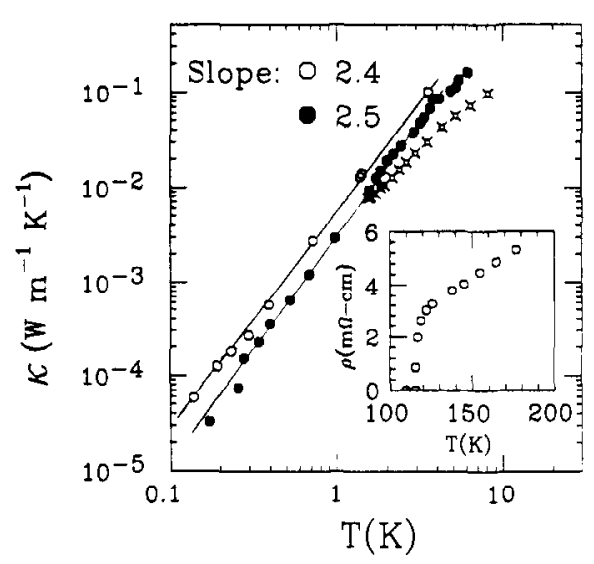

Fig. 2. Thermal conductivity of $\mathrm{Tl}_{2} \mathrm{Ba}_{2} \mathrm{Ca}_{2} \mathrm{Cu}_{3} \mathrm{O}_{10}$ at low temperatures. The data of Castello et al. [8] are shown by stars. The inset shows the electrical resistivity for the sample whose thermal conductivity is designated by open circles. 
In contrast, it is interesting to note that the low-temperature data of Castello et al. (down to $1.5 \mathrm{~K}$ ) deviate from the $T^{2}$ variation in the opposite direction, namely their $\kappa(T)$ dependence is subquadratic. It is not clear why the thermal conductivity of Tland Bi-based ceramics differs so much from the $Y$ based perovskites. Perhaps the reason lies in the fact that neither $\mathrm{Tl}-\mathrm{Ba}-\mathrm{Ca}-\mathrm{Cu}-\mathrm{O}$ nor $\mathrm{Bi}-\mathrm{Sr}-\mathrm{Ca}-\mathrm{Cu}-\mathrm{O}$ ceramics are single-phase materials. It would be very useful to measurc the thermal conductivity on single crystals of $\mathrm{Tl}-\mathrm{Ba}-\mathrm{Ca}-\mathrm{Cu}-\mathrm{O}$. Such measurements should reveal the intrinsic behaviour of the material and allow for a detailed comparison with other high$T_{\mathrm{c}}$ superconductors. Although some progress has been made recently [23] in the preparation of single crystals of this family of perovskites, sufficiently large specimens suitable for thermal transport studies are not yet available.

\section{Acknowledgement}

This work was supported by US Army Research Office Contract No. DAAL-03-87-K-0007.

\section{References}

[1] Z.Z. Sheng and A.M. Hermann, Nature 332 (1988) 55.

[2] C.C. Torardi, M.A. Subramanian, J.C. Calabrese, J. Gopalakrishnan, E.M. McCarron, K.J. Morrissey, T.R. Askew, R.B. Flippen, U. Chowdhury and A.W. Sleight, Phys. Rev. B 38 ( 1988) 225.

[3] S.S.P. Parkin, V.Y. Lee, A.I. Nazzal, R. Savoy, R. Bcyers and S.J. La Placa, Phys. Rev. Lett. 61 (1988) 750.

[4] H. Ihara, R. Sugise, M. Hirabayashi, T. Terada, M. Jo, K. Hayashi, M. Tokumoto, Y. Kimura and T. Shimomura, Nature 334 (1988) 510.
[5] P.T. Wu, R.S. Liu, J.M. Liang, W.H. Lee, L. Chang, L.J. Chen and C.T. Chang, Physica C 156 (1988) 109.

[6] C. Uher, J. Supercond. 3 (1990) 337.

[7] F.G. Aliev, V.V. Moshchalkov and V.V. Pryadun, Physica C 162-164 (1989) 572.

[8] D. Castello, M. Jaime, M. Nunez Regueiro and C. Fainstein, in: Progress in High Temperature Superconductivity, vol. 25, ed. R. Nicolsky, (World Scientific Publishing, Singapore, 1990) p. 452.

[9] C. Uher, in: Superconductivity and Applications, eds. H.S. Kwok et al. (Plenum, NY, 1990) p. 217.

[10] V. Florentiev, A. Inyushkin, A. Taldenkov, O. Melnikov and A. Bykov, in: Progress in High Temperature Superconductivity, vol. 25, ed. R. Nicolsky, (World Scientific Publishing, Singapore, 1990) p. 462.

[11] S.D. Peacor, J.L. Cohn and C. Uher, Phys. Rev. B 43 (1991).

[12] A. Jezowski, A.J. Zaleski, H. Misiorek, E.P. Khlybov and V.V. Evdokimova, Phys. Lett. A 139 (1989) 265.

[13] D.T. Morelli, G.L. Doll, J. Heremans, S.D. Peacor, C. Uher, M.S. Dresselhaus, A. Cassanho, D.R. Gabbe and H.P. Jenssen, Solid State Commun. 77 (1991) 773.

[14] M. Nunez Regueiro, D. Castello, M.A. Izbizky, E. Esparza and C.D. D'Ovidio, Phys. Rev. B 36 (1987) 8813.

[15] W.A. Phillips, J. Low-Temp. Phys. 7 (1972) 351.

[16] P.W. Anderson, B.I. Halperin and C.M. Varma, Philos. Mag. 25 (1972) 1.

[17] D.M. Zhu, A.C. Anderson, E.D. Bukowski and D.M. Ginsberg, Phys. Rev. B 40 (1989) 841.

[18] G. Sparn, M. Baenitz, S. Horn, F. Steglich, W. Assmus, T. Wolf, A. Kapitulnik and Z.X. Zhao, Physica C. 162-164 (1989) 508.

[19] S.D. Peacor and C. Uher, Phys. Rev. B 39 (1989) 11559.

[20] U. Gottwick, R. Held, G. Sparn, F. Steglich, H. Rietschel, D. Ewert, B. Renker, W. Bauhoffer, S. von Molnar, M. Wilhelm and H.E. Hoenig, Europhys. Lett. 4 (1987) 1183.

[21 ] J.E. Graebner, L.F. Schneemeyer, R.J. Cava, J.V. Waszczak and E.A. Rietman, Symp. Proc. Mater. Res. Soc. 99 (1988) 745.

[22] J.L. Cohn, S.D. Peacor and C. Uher, Phys. Rev. B 38 (1988) 2892.

[23] L. Shu-Yuan, L. Li, Z. Dian-Liu, H.-M. Duan and A.M. Hermann, Europhys. Lett. 12 (1990) 641. 